\title{
Disease activity and treatment patterns in rheumatoid arthritis in Qatar: Data from the Qatar rheumatoid arthritis registry
}

Introduction/Objectives: To describe disease activity and treatment patterns in patients with rheumatoid arthritis in Qatar. Methods: Registry data from consecutive patients who attended routine rheumatology outpatient clinic appointments at the Hamad General Hospital between June 2013 and September 2015 were evaluated. Results: Data on 496 patients (female, 75.8\%) were analyzed: 75.6\% were rheumatoid factor positive, $79.6 \%$ were anti-cyclic citrullinated peptide positive, and $30.6 \%$ had erosive arthritis at data collection. Methotrexate (MTX) was the most commonly prescribed synthetic DMARD (in 65.3\%): $27 \%$ monotherapy, $20.8 \%$ combined with hydroxychloroquine or sulfasalazine or leflunomide, and $4.2 \%$ triple therapy. Hydroxychloroquine, sulfasalazine, or leflunomide were used by $29.4 \%, 21.2 \%$, and $13.7 \%$ of patients, respectively; $<5 \%$ of patients used these drugs as monotherapy. One quarter of patients (26.4\%) were receiving biologic drugs (etanercept, $9.3 \%$; rituximab, $6 \%$; tocilizumab, $5.6 \%$; adalimumab, $4.4 \%$; abatacept, $0.4 \%$; certolizumab, $0.4 \%$; infliximab, $0.2 \%$ ). Among patients who were receiving biologic therapy, $32 \%$ were on monotherapy. Patients' disease activity status was found to vary according to the employed measure of disease activity, with greater proportions of patients in remission reported using the Disease Activity Score 28 (30.3\%) vs. Clinical Disease Activity Index (18\%) and the Simple Disease Activity Index (17.5\%). Conclusion: Treatment patterns in RA patients in Qatar are similar to those reported in registries worldwide. Therapy commonly includes MTX with an increasing number of patients being on biologic medications. Disease activity status varies according to methodology.

Keywords: rheumatoid arthritis • anticcp • rheumatoid factor • synthetic dMARD • biologic dMARD

\section{Introduction}

Rheumatoid Arthritis (RA) is a chronic inflammatory condition characterized by swollen, painful joints, leading to permanent joint destruction if not treated early and appropriately $[1,2]$. High disease activity $[1,3]$, disease duration [4], and joint destruction $[1,3]$ contribute to the significant and progressive increase in disability and reduction in function.

Advances in understanding of the pathogenetic pathways in RA have driven the continuous development and introduction of new classes of treatments to our once-limited repertoire of therapeutic options [5]. The treatment paradigm has been shifted from analgesics and non-steroidal anti-inflammatory drugs to more effective Disease-Modifying Anti-Rheumatic Drugs (DMARDs). Patient outcomes have significantly improved through the safe and effective use of methotrexate (MTX) as well as better access to biological agents [5]. They have also gained from awareness of the benefits of early treatment and a treatment target of disease remission or low disease activity $[5,6]$. As such, current recommendations for the therapeutic approach to RA are to start treatment with DMARDs as soon as the diagnosis is made, and to aim treatment toward reaching a target of remission or low disease activity, to avoid permanent disability [6].

MTX is the preferred initial DMARD after clinical diagnosis in most cases. The efficacy of MTX in the treatment of RA has been demonstrated in observational studies and randomized controlled trials, and established over the long term [7]. Other conventional synthetic DMARDs (csDMARDs), including hydroxychloroquine [8], sulfasalazine [9], and leflunamide [10] have also been successfully used in RA patients and remain important therapies [5]. However, because MTX is the most extensively studied drug it is usually used as a first line unless contraindicated [6].

Combination therapy with DMARDs is indicated in patients who fail monotherapy and have persistent disease activity. MTX is also highly effective in combination with other DMARDs [5], including csDMARDs [11,12] and csDMARDs with biologic DMARDs

\author{
Fiaz Alam*, Mohammed \\ Hammoudeh, Hani Malallah \\ Abdulaziz, Housam Aldeen \\ Sarakbi, Abdul Rahim \\ Mohammed Siam, Salah \\ Mohamed Mehdi, Izzat Ali \\ Mohamed Khanjar, Nabeel \\ Abdulla, Abdul Razzakh Poil, \\ Abdo Qaid Lutf, Eman Hassan \\ Satti El Sayed, Shaymaa EI \\ Atwy \& Samar Al Emadi* \\ Department of Rheumatology, Hamad \\ General Hospital, Doha, Qatar \\ *Author for correspondence: \\ falam1@hamad.qa \\ salemadi@hamad.qa
}


(bDMARDs) [12-16]. bDMARDs that specifically target factors known to play important roles in RA pathology have further advanced the treatment of RA: Tumor necrosis factor (TNF) inhibitors [12-14] and a variety of other biologic agents with different targets $[15,16]$ have shown efficacy against RA. bDMARDs are generally recommended in patients with poor response to csDMARDs [6].

Different RA guidelines and recommendations have focused on DMARDs followed by a treatto-target approach to manage this chronic debilitating disease [2,6]. While different options are available, in real-life practice, the choice of treatment may vary to some extent in different parts of the world. Choice of treatment may depend on patients' tolerability and other medical conditions, local practice, availability of therapies, cost, and patients' preferences.

The RA registry at the Hamad General Hospital in Qatar was established in 2013, and is the first of its kind to study patient characteristics and treatment use and response in the Middle East. The aim of the registry is to collect reallife continuous data on patients with RA seen at routine clinic visits. In this prospective, longitudinal, observational study of data from the registry, we report the demographics, treatment, and outcomes in patients with RA living in Qatar.

\section{Methods}

\section{Patients}

The Qatar RA registry was accessed to collect data from all patients aged 16-75 years attending routine rheumatology outpatient clinic appointments at the Hamad General Hospital, Doha, Qatar. (Hamad General Hospital was the single biggest tertiary hospital till 2015, receiving majority of rheumatology referral across the country). The RA registry collects data only on patients who fulfil the 2010 American College of Rheumatology (ACR) criteria for RA.

Data from consecutive patients who attended rheumatology outpatient clinic appointments between June 2013 and September 2015 were analyzed. All consecutive patients identified by the treating rheumatologist during their routine outpatient clinic visits in Hamad General Hospital, were referred to the research team after fulfilment of ACR criterial for RA. All patients provided signed informed consent to participate and patients without informed consent were excluded from the study. The study was approved by the Institutional Review Board of the Hamad Medical Corporation, Doha, Qatar.

\section{Data capture and analysis}

Demographic data (age, gender), comorbidities (diabetes, hypertension, cardiovascular, diseases, dyslipidemia thyroid disorders and osteoporosis), smoking status, information about disease onset and duration, and type and side effects of medications were recorded on predefined questionnaire. Data regarding rheumatoid factor and anti-cyclic citrullinated peptide (CCP) status, inflammatory markers (erythrocyte sedimentation rate and C-reactive protein), and radiographic images were collected from electronic medical records. All treatments as prescribed by attending clinicians were recorded on electronic medical records after inquiring the patients.

Disease activity and functional ability scores were measured by treating rheumatologist, at each visit using the Disease Activity Score 28 Joints (DAS28), Clinical Disease Activity Index (CDAI), Simple Disease Activity Index (SDAI), and Health Assessment Questionnaire (HAQ) was recorded by the research assistant. Scores were calculated and recorded for each visit.

\section{Statistical analysis}

Following extraction, data was evaluated for completeness. Descriptive analyses, including means, standard deviations (SD), minimum and maximum for continuous variables and frequencies and percentages for categorical variables, were used to summarize the patient's demographic and clinical characteristics, treatment patterns, and disease activity scores. All statistics were performed using SPSS 22 (SPSS Inc. Chicago, IL).

\section{Results}

\section{Patient demographic and disease} characteristics

Data on 496 patients (female, $n=376 ; 75.8 \%$ ) were entered onto the RA registry database during the study period. Rheumatoid factor was positive in $370(74.9 \%)$ and anti-CCP in 395 (79.8\%) patients. Approximately one third of patients $(n=152 ; 31.3 \%)$ had erosive arthritis (Table 1).

\section{Treatment patterns}

MTX was the most commonly prescribed synthetic DMARD (65.3\%), with $27 \%$ of patients receiving MTX as monotherapy, $20.8 \%$ receiving MTX combined with hydroxychloroquine, sulfasalazine, or leflunomide, and 4.2\% MTX as triple therapy 


\begin{tabular}{|c|c|}
\hline Characteristic & Patients n (\%) \\
\hline Total, N & 496 \\
\hline Arab & $269(54.2)$ \\
\hline Qatari & $117(23.6)$ \\
\hline Non-Qatari & $152(30.6)$ \\
\hline Non-Arab & $227(45.8)$ \\
\hline Asian & $198(39.9)$ \\
\hline Other & $29(5.9)$ \\
\hline \multicolumn{2}{|l|}{ Sex } \\
\hline Male & $120(24.2)$ \\
\hline Female & $376(75.8)$ \\
\hline Rheumatoid factor positive & $370 / 494(74.9)$ \\
\hline Anti-CCP positive & $395 / 495(79.8)$ \\
\hline Erosive arthritis & $152 / 485(31.3)$ \\
\hline Seronegative arthritis & $74(14.9)$ \\
\hline \multicolumn{2}{|c|}{ CCP, cyclic citrullinated peptide } \\
\hline
\end{tabular}

(Table 2). MTX was used in combination with bDMARDs by $13.3 \%$ of patients. Less than $5 \%$ of patients were receiving hydroxychloroquine, sulfasalazine, or leflunomide as monotherapy. Less than half patients $(39.7 \%)$ had received corticosteroids.

The mean \pm standard deviation (SD) dose of MTX, sulfasalazine, and prednisolone was 15.9 $\pm 5.0 \mathrm{mg} /$ week, $1.6 \pm 0.6 \mathrm{~g} / \mathrm{day}$, and $5.5 \pm 1.8$ $\mathrm{mg} /$ day, respectively.

Approximately one quarter $(26.4 \%)$ of patients were receiving bDMARDs (Table 3). Etanercept was most frequently used $(9.3 \%)$, followed by rituximab, tocilizumab, and adalimumab; only $1 \%$ were treated with abatacept, certolizumab, or infliximab. Similar numbers of patients took tumor necrosis factor (TNF) inhibitors and nonTNF inhibitor biologics. One third (32.1\%) of patients were receiving bDMARD monotherapy, mostly rituximab or tocilizumab. Half $(50.4 \%)$ patients took bDMARD in combination with MTX.

\section{Disease activity scores}

Mean \pm SD disease and function outcomes scores among the study cohort were: $3.2 \pm 1.2$ for DAS28; $8.5 \pm 7.9$ for CDAI; $9.7 \pm 9.1$ for SDAI; and $0.9 \pm 0.7$ for HAQ.

Patients' disease activity status was found to vary according to employed measure of disease activity. Thus similar proportions of patients were reported in remission (defined as $\mathrm{SDAI} \leq 3.3$ and CDAI $\leq 2.8)$ using CDAI $(18 \%)$ and SDAI

\begin{tabular}{|c|c|}
\hline $\begin{array}{c}\text { Total,N } \\
\text { Synthetic cDMARDs }\end{array}$ & $\begin{array}{c}\text { Patients } \\
496 \\
n(\%)\end{array}$ \\
\hline MTX & $324(65.3)$ \\
\hline MTX monotherapy & $134(27.0)$ \\
\hline $\mathrm{MTX}+\mathrm{HCQ}$ & $49(9.9)$ \\
\hline MTX + SSZ & $30(6.1)$ \\
\hline MTX + LFM & $24(4.8)$ \\
\hline MTX + bDMARD & $66(13.3)$ \\
\hline $\mathrm{MTX}+\mathrm{SSZ}+\mathrm{HCQ}$ & 18 (3.6) \\
\hline $\mathrm{MTX}+\mathrm{LFM}+\mathrm{HCQ}$ & $3(0.6)$ \\
\hline $\mathrm{HCQ}$ & $146(29.4)$ \\
\hline HCQ monotherapy & $23(4.6)$ \\
\hline $\mathrm{HCQ}+\mathrm{SSZ}$ & $24(4.8)$ \\
\hline $\mathrm{HCQ}+\mathrm{LFM}$ & $6(1.2)$ \\
\hline SSZ & $105(21.2)$ \\
\hline SSZ monotherapy & $16(3.2)$ \\
\hline SSZ + LFM & $7(1.2)$ \\
\hline LFM & $68(13.7)$ \\
\hline LFM monotherapy & $17(3.4)$ \\
\hline \multicolumn{2}{|c|}{$\begin{array}{l}\text { bDMARD, biologic disease-modifying anti-rheumatic } \\
\text { drug; cDMARD, conventional disease-modifying } \\
\text { anti-rheumatic drug; HCQ, hydroxychloroquine; LFM, } \\
\text { leflunomide; MTX, methotrexate; SSZ, sulfasalazine. }\end{array}$} \\
\hline
\end{tabular}

(17.5\%) scores, whereas a higher proportion of patients $(30.3 \%)$ were in remission (defined as DAS28 ESR <2.6) according to their DAS28 scores. Reflecting this, low disease activity status (DAS28 ESR 2.6-3.2, SDAI $\leq 11$, CDAI $\leq 10$ ) was recorded for $23.1 \%$ of patients as evaluated by DAS28 score and $51.6 \%$ of patients according to their CDAI and SDAI scores.

\section{Discussion}

The findings of this study in patients enrolled in the Qatar RA database reflect the general RA treatment patterns practiced worldwide. In the Qatar registry, almost two thirds of patients were using MTX either alone or in combination with other DMARDS, and more than one quarter were receiving biologic DMARDS including TNF inhibitors and non-TNF inhibitor biologics. Approximately one third of patients on a bDMARD took this as monotherapy. This study contributes to the scarce data that are available regarding RA treatment patterns from Arab populations.

Other studies of Arab populations indicate 


\begin{tabular}{|c|c|}
\hline Total, N & $\begin{array}{l}\text { Patients } \\
\text { N=496 } \\
\text { n (\%) }\end{array}$ \\
\hline bDMARDs & $131(26.4)^{*}$ \\
\hline TNF inhibitors & $71(14.3)$ \\
\hline Etanercept & $46(9.3)$ \\
\hline Adalimumab & $22(4.4)$ \\
\hline Certolizumab & $2(0.4)$ \\
\hline Infliximab & $1(0.2)$ \\
\hline Non-TNF inhibitors & $60(12.1)$ \\
\hline Rituximab & $30(6.1)$ \\
\hline Tocilizumab & $28(5.6)$ \\
\hline Abatacept & $2(0.4)$ \\
\hline bDMARD monotherapy & $42(32.1) \dagger$ \\
\hline TNF inhibitors & $16(12.2)$ \\
\hline Etanercept & $9(6.9)$ \\
\hline Adalimumab & $7(5.3)$ \\
\hline Non-TNF inhibitors & $26(19.8)$ \\
\hline Rituximab & $13(9.9)$ \\
\hline Tocilizumab & $13(9.9)$ \\
\hline \multicolumn{2}{|c|}{$\begin{array}{l}\text { *Denominator = total no. patients on RA treatment } \\
(\mathrm{N}=496) . \\
\text { tDenominator = total no. on bDMARD treatment } \\
(\mathrm{N}=131) \text {. } \\
\text { bDMARD, biologic disease-modifying anti-rheumati } \\
\text { drug; RA, rheumatoid arthritis; } \mathrm{TNF} \text {, tumor necrosis } \\
\text { factor. }\end{array}$} \\
\hline
\end{tabular}

somewhat higher rates of MTX use than that found in our study. MTX was used at RA disease onset in $89.3 \%$ of Egyptian patients aged 16-40 years and $58.4 \%$ of patients over 60 years old [17]. Studies from Saudi Arabia [18,19] and Jordan [20] found that MTX was used in 71\%$75 \%$ of RA patients. In Saudi Arabia, as in our study, hydroxychloroquine was the second most frequently used csDMARD (34\%), whereas use of sulfasalazine $(3.4 \%)$ and leflunomide $(2.4 \%)$ was considerably less frequent [19].

A higher percentage of RA patients in a Saudi Arabian cohort [18] were on bDMARDs
$(41.5 \%)$ as compared to our cohort, whereas only $24 \%$ were on bDMARDs (TNF inhibitors) in Jordan [20]. Across northern Algeria, 89.7\% of RA patients were taking DMARDs and only 4\% were taking bDMARDs (rituximab) [21].

MTX is the preferred initial DMARD recommended in the USA and Europe [2,6]. It is highly effective when used as monotherapy or in combination with glucocorticoids, other csDMARDs, and bDMARDs [6,7]. MTX as anchor drug in RA management $[6,7]$ is reflected in the majority of studies worldwide. In a multicenter RA registry in Japan [22] and the Danish DANBIO register [23], MTX use among biologic-naïve patients was $74.9 \%$ and $76 \%$, respectively.

Use and type of non-MTX csDMARDs depend on local rheumatology practice and guidelines, and availability. csDMARDs that are commonly used include hydroxychloroquine, sulfasalazine, and leflunomide. The EULAR Study Group for Registers and Observational Drug Studies reported that the use of non-MTX csDMARDs ranged from $8 \%$ in Norway to $65 \%$ in Finland [24]. Compared with MTX, we found fewer patients were taking other csDMARDs as monotherapy in our cohort. A similar finding was observed in 232 patients with recentonset RA in the US ERATER registry, where csDMARD monotherapy comprised MTX in $81.5 \%$ of patients, hydroxychloroquine in $6.5 \%$, sulfasalazine in $0.9 \%$, and leflunomide in $2.6 \%$ [25].

MTX as monotherapy was prescribed in $27 \%$ of our cohort compared with $35.3 \%$ of a Norwegian cohort [26]. However, sulfasalazine, leflunamide, or either drug in a triple therapy regimen was less frequently prescribed in this Norwegian cohort than in our study (sulfasalazine $9.9 \%$ vs. $21.2 \%$; leflunamide $11 \%$ vs. $13.7 \%$; triple therapy $2 \%$ vs. $4.2 \%$, respectively).

It is generally considered that MTX in combination with bDMARDs has a superior effect than either MTX or the bDMARD alone in the majority of cases. We report $13.3 \%$ of patients were treated with MTX in combination with bDMARDs in Qatar. Furthermore, almost half of patients taking bDMARD were receiving concomitant MTX. In comparison, in European registries use of concomitant MTX plus bDMARD ranged from $41 \%$ in Switzerland to $91 \%$ in Slovenia [24]. High rates have been reported in diverse areas such as China [27] and Australia [28] where combination therapy with 
MTX and bDMARDs accounted for $65.9 \%$ and $66.3 \%$, respectively. MTX as part of a combination therapy was reported in $68.6 \%$ of biologic-naïve patients in the US Corrona registry [29] and in $78.7 \%$ of patients switching from first-line TNF inhibitor therapy in the Japanese Tsurumai Biologics Communication registry [30]. In the Dutch DREAM registry, almost half of patients were receiving a TNF inhibitor with MTX and $16.6 \%$ a TNF inhibitor as monotherapy [31].

Real-life registry studies from different countries indicate that some patients continue to receive bDMARDs as monotherapy. A comparative analysis between bDMARD monotherapy and combination therapy with csDMARDs in the Czech Attra RA registry demonstrated that $12 \%$ of patients were using bDMARDs as monotherapy and that of those patients $61.4 \%$ remained on the same biologic monotherapy after 12 months [32]. In Denmark, one in five RA patients (19\%) were prescribed bDMARDs as monotherapy of which etanercept $(36.6 \%)$, adalimumab (21.3\%), and tocilizumab (15.3\%) were the most frequently prescribed agents [33].

The EULAR Study Group for Registers and Observational Drug Studies showed that the rates of use of TNF inhibitors varied considerably [24]. Adalimumab was used in from $9 \%$ of RA patients in Norway to $97 \%$ in the UK, etanercept from $28 \%$ in Denmark to $65 \%$ in Norway, infliximab from $6 \%$ in The Netherlands to $38 \%$ in Denmark, and certolizumab and golimumab in $1 \%$ each in Germany. Other bDMARDs were used less frequently; rituximab from $2 \%$ in The Netherlands to $14 \%$ in Finland, tocilizumab (10\% in Germany), and abatacept (3\% in Switzerland). Similar to our database, etanercept followed by adalimumab was the most frequently used bDMARD in Australian (60.7\% and 33.4\%) [28] and Danish (28\% and 21\%) [23] registries. Conversely, the bDMARD of first choice was infliximab in an Italian biologics registry $38.1 \%$ of patients vs. etanercept, $30.7 \%$ and adalimumab, $31.2 \%$ ) [34] and in the Danish DANBIO registry $(59 \%$ vs. etanercept, $18 \%$ and adalimumab, 23\%) [35].

While the majority of DMARD prescriptions are initiated as combination therapy, monotherapy is also commonly used in RA patients [36]. A Swiss registry found that once initiated, monotherapy is continued during the entire treatment course in $86 \%$ of patients (vs. $87 \%$ of patients initiated on combination therapy) [36]. In our cohort one third of patients were initiated on bDMARD monotherapy, similarly to an Argentinian registry that reported $35 \%$ of biologics were initiated as monotherapy [37]. In the US Corrona registry, a bDMARD was initiated as monotherapy in $19.1 \%$ of biologic-naïve patients [29]. TNF inhibitor monotherapy was used in $12.2 \%$ of patients in our cohort as compared with $8.4 \%$ in a Norwegian cohort of RA patients [26].

Rituximab and tocilizumab were used in less than $10 \%$ of patients in our database. The French ORO registry reported $33.3 \%$ of patients received abatacept as monotherapy, with abatacept administered as the first bDMARD in $12 \%$ of cases [38]. Rituximab monotherapy was used by $23 \%$ of patients in the US Corrona Registry [39], and $35.3 \%$ of patients started on tocilizumab monotherapy in the Swedish biologics registry [40]. Data from ten different registries with information on tocilizumab use revealed that tocilizumab was most frequently started in combination with MTX, followed by tocilizumab as monotherapy, tocilizumab with csDMARDs (not MTX), and tocilizumab in combination with MTX and other csDMARD(s) [41].

In Canada the rate of corticosteroid use was $30 \%-40 \%$, with up to three quarters of patients exposed at some time during their treatment [42]. Corticosteroids were also used by approximately $40 \%$ of patients in our study, but use varies considerably across the Middle East $(29.3 \%-80.8 \%)[17,19,20]$. In northern Algeria, more than $90 \%$ of patients were taking glucocorticoids [21].

Worldwide, registries indicate that there is significant variation in the use of DMARDs for the treatment of RA. Despite MTX being considered as the anchor drug for RA treatment $[6,7]$, either as monotherapy or in combination therapy, a proportion of patients are prescribed other DMARDs as monotherapy. Medication use is generally guided by local practice guidelines, availability, and cost. However, variations may also reflect the different methods of data collection and patient recruitment criteria, such as those of prospective studies with predefined parameters and those from routine clinic visits. Patients may be starting their first DMARD or starting another treatment following DMARD failure.

Greater proportions of patients in our cohort were in remission compared with RA cohorts from other parts of the Arab world. Alawneh et 
al. [20] reported that $5 \%$ of Jordanian patients were in remission when DAS28 was used as disease activity measure whereas only $1 \%$ were in remission when CDAI was employed. DAS28 (ESR) is less stringent as reported by Fujiwara and Kita [43]. Indeed, Medeiros et al. [44] reported that $15.6 \%$ of patients were in DAS28 (ESR) remission whereas only $4.2 \%$ were in remission if CDAI or SDAI was used as disease activity measure. Similar trends were observed in our cohort of patients.

Our study has various limitations. First, although, the data is from single center but it was the only governmental tertiary hospital in the country receiving majority of rheumatology referral apart from one secondary center. Second, the study design is observational but it explains the real life data of rheumatoid arthritis patients from this part of world which is not reported before. Third, there was missing data about timing of initiation of various treatment including biological treatment, follow up disease activity scores and some of the patients lost follow up as well. Due to these reasons it was difficult to draw any association between given treatment and disease activity scores.

\section{Conclusion}

In conclusion, the aim of this study was to determine common RA therapies prescribed to patients in Qatar. The Qatar registry substantiates that MTX alone or in combination is used as an anchor drug in the treatment of RA, and that one third of patients are prescribed bDMARDs as monotherapy. For contextualization, we compared our registry data with those reported in other parts of the world, and found that treatment patterns in RA patients in Qatar are similar to those reported in registries worldwide.

\section{References}

1. Breedveld FC, Han C, Bala M, et al. Association between baseline radiographic damage and improvement in physical function after treatment of patients with rheumatoid arthritis. Ann. Rheum. Dis. 64(1), 52-55 (2005).

2. Aletaha D, Neogi T, Silman AJ, et al. Rheumatoid arthritis classification criteria: An American college of rheumatology/European League against rheumatism collaborative initiative. Ann. Rheum. Dis. 69, 15801588 (2010).

3. Bombardier C, Barbieri M, Parthan A, et al. The relationship between joint damage and functional disability in rheumatoid arthritis: A systematic review. Ann. Rheum. Dis. 71(6), 836-844 (2012).

4. Molenaar ET, Voskuyl AE, Dijkmans BA. Functional disability in relation to radiological damage and disease activity in patients with rheumatoid arthritis in remission. J. Rheumatol. 29, 267-270 (2002).

5. McInnes IB, O'Dell JR. State-of-the-art: Rheumatoid arthritis. Ann. Rheum. Dis. 69(11), 1898-1906 (2010).

6. Smolen JS, Landewé R, Breedveld FC, et al. EULAR recommendations for the management of rheumatoid arthritis with synthetic and biological disease-modifying antirheumatic drugs: 2013 update. Ann. Rheum. Dis. 73(3), 492-509 (2014)

7. Pincus T, Yazici Y, Sokka T, et al. Methotrexate as the "anchor drug" for the treatment of early rheumatoid arthritis. Clin. Exp. Rheumatol. 21(Suppl 31), S178185 (2003).

8. Pavelka K Jr, Sen KP, Pelísková Z, et al. Hydroxychloroquine sulphate in the treatment of rheumatoid arthritis: A double blind comparison of two dose regimens. Ann. Rheum. Dis. 48(7), 542-546 (1989).

9. Weinblatt ME, Reda D, Henderson W et al. Sulfasalazine treatment for rheumatoid arthritis: a metaanalysis of 15 randomized trials. J. Rheumatol. 26, 2123-2130 (1999)

10. Maddison P, Kiely P, Kirkham B, et al. Leflunomide in rheumatoid arthritis: Recommendations through a process of consensus. Rheumatology (Oxford). 44(3), 280-286 (2005).

11. Dale J, Alcorn N, Capell H, et al. Combination therapy for rheumatoid arthritis: Methotrexate and sulfasalazine together or with other DMARDs. Nat. Clin. Pract. Rheumatol. 3, 450-458 (2007).

12. Moreland LW, O'Dell JR, Paulus HE, et al. A randomized comparative effectiveness study of oral triple therapy versus etanercept plus methotrexate in early aggressive rheumatoid arthritis: The treatment of early aggressive rheumatoid arthritis trial. Arthritis. Rheum. 64(9), 2824-2835 (2012).

13. Breedveld FC, Weisman MH, Kavanaugh AF, et al. The PREMIER study- A multicenter, randomized, double-blind clinical trial of combination therapy with adalimumab plus methotrexate versus methotrexate alone or adalimumab alone in patients with early, aggressive rheumatoid arthritis who had not had previous methotrexate treatment. Arthritis. Rheum. 54(1), 26-37 (2006).

14. St Clair EW, van der Heijde DM, Smolen JS, et al. Combination of infliximab and methotrexate therapy for early rheumatoid arthritis: a randomized, controlled trial. Arthritis. Rheum. 50(11), 3432-3443 (2004).

15. Tak PP, Rigby WF, Rubbert-Roth A, et al. Inhibition of joint damage and improved clinical outcomes with rituximab plus methotrexate in early active rheumatoid arthritis: the IMAGE trial. Ann. Rheum. Dis. 70(1), 39-46 (2011).

16. Emery P, Keystone E, Tony HP, et al. IL-6 receptor inhibition with tocilizumab improves treatment outcomes in patients with rheumatoid arthritis refractory to anti-tumour necrosis factor biologicals: results from a 24-week multicentre randomised placebo-controlled trial. Ann. Rheum. Dis. 67(11), 1516-1523 (2008)

17. El-Labban AS, Omar HA, El-Shereif RR, et al. Pattern of young and old onset rheumatoid arthritis (YORA and EORA) among a group of Egyptian patients with rheumatoid arthritis. Clin. Med. Insights. Arthritis. Musculoskelet. Disord. 3, 25-31(2010).

18. Al-Bishri J, Attar S, Bassuni N, et al. Comorbidity 
profile among patients with rheumatoid arthritis and the impact on prescriptions trend. Clin. Med. Insights. Arthritis. Musculoskelet. Disord. 6, 11-18 (2013).

19. Safi M-A A, Fathadin OA. Pattern of drugs use and association with anti-mutated citrullinated vimentin antibody in rheumatoid arthritis. Saudi. Med. J. 36(4), 316-323 (2015).

20. Alawneh KM, Khassawneh BY, Ayesh MH, et al Rheumatoid arthritis in Jordan: a cross sectional study of disease severity and associated comorbidities. Ther. Clin. Risk. Manag. 10, 363-366 (2014).

21. Slimani S, Abbas A, Ben Ammar A, et al. Characteristics of rheumatoid arthritis in Algeria: a multicenter study. Rheumatol. Int. 34(9), 1235-1239 (2014).

22. Kojima T, Kaneko A, Hirano Y, et al. Study protocol of a multicenter registry of patients with rheumatoid arthritis starting biologic therapy in Japan: Tsurumai Biologics Communication Registry (TBCR) study. Mod. Rheumatol. 22(3), 339-345 (2012).

23. Hetland ML, Jensen DV, Krogh NS. Monitoring patients with rheumatoid arthritis in routine care: experiences from a treat-to-target strategy using the DANBIO registry. Clin. Exp. Rheumatol. 32(5 Suppl 85), S141-146 (2014).

24. Kearsley-Fleet L, Závada J, Hetland ML. The EULAR study group for registers and observational drug studies: comparability of the patient case mix in the European biologic disease modifying anti-rheumatic drug registers. Rheumatology (Oxford). 54(6), 10741079 (2015).

25. Sokka T, Pincus T. An early rheumatoid arthritis treatment evaluation registry (ERATER) in the United States. Clin. Exp. Rheumatol. 23(5 Suppl 39), S178181 (2005).

26. Kvien TK, Heiberg MS, Lie E, et al. A Norwegian DMARD register: Prescriptions of DMARDs and biological agents to patients with inflammatory rheumatic diseases. Clin. Exp. Rheumatol. 23(5 Suppl 39), S188-194 (20050.

27. An Y, Liu T, He D, et al. The usage of biological DMARDs and clinical remission of rheumatoid arthritis in China: A real-world large scale study. Clin. Rheumatol. 36(1), 35-43 (2017).

28. Buchbinder R, March L, Lassere M, et al. Effect of treatment with biological agents for arthritis in Australia: The Australian rheumatology association database. Intern. Med. J. 37(9), 591-600 (2007).

29. Pappas DA, Reed GW, Saunders K. Characteristics associated with biologic monotherapy use in biologicnaive patients with rheumatoid arthritis in a US registry population. Rheumatol. Ther. 2(1), 85-96 (2015).

30. Hirabara S, Takahashi N, Fukaya N, et al. Clinical efficacy of abatacept, tocilizumab, and etanercept in Japanese rheumatoid arthritis patients with inadequate response to anti-TNF monoclonal antibodies. Clin. Rheumatol. 33(9), 1247-1254 (2014).

31. Manders SH, Kievit W, Jansen TL, et al. Effectiveness of tumor necrosis factor inhibitors in combination with various csDMARD in the treatment of rheumatoid arthritis: Data from the DREAM registry. J. Rheumatol. 43(10), 1787-1794 (2016).

32. Mann H, Forejtová Š, Jarošová K. Monotherapy with biological disease-modifying anti-rheumatic drugsdata from the Czech registry Attra. Ann. Rheum. Dis. 73(Suppl 2), 238 (2014).
33. Jørgensen TS, Kristensen LE, Christensen R Effectiveness and drug adherence of biologic monotherapy in routine care of patients with rheumatoid arthritis: a cohort study of patients registered in the Danish biologics registry. Rheumatology (Oxford). 54, 2156-2165 (2015).

34. Favalli EG, Pregnolato F, Biggioggero M, et al. Twelveyear retention rate of first-line tumor necrosis factor inhibitors in rheumatoid arthritis: real-life data from a local registry. Arthritis. Care. Res. (Hoboken) 68(4), 432-439 (2016).

35. Ørnbjerg LM, Østergaard M, Bøyesen P, et al. Which factors influence radiographic progression during treatment with tumor necrosis factor inhibitors in clinical practice? Results from 930 patients with rheumatoid arthritis in the nationwide Danish DANBIO registry. J. Rheumatol. 41(12), 2352-2360 (2014).

36. Gabay C, Riek M, Scherer A, et al. (2015) Effectiveness of biologic DMARDs in monotherapy versus in combination with synthetic DMARDs in rheumatoid arthritis: Data from the swiss clinical quality management registry. Rheumatology (Oxford). 54(9), 1664-1672.

37. Catay E, Bravo M, Rosa J, et al. Prevalence of biologics monotherapy in a cohort of patients with rheumatoid arthritis in daily clinical practice. BMC. Musculoskelet. Disord. 17(1), 110 (2016).

38. Truchetet ME, Poursac N, Barnetche T, et al. Abatacept monotherapy compared with abatacept plus diseasemodifying anti-rheumatic drugs in rheumatoid arthritis patients: Data from the ORA registry. Arthritis. Res. Ther. 18(1), 72 (2016).

39. Harrold LR, Reed GW, Shewade A. Effectiveness of rituximab for the treatment of rheumatoid arthritis in patients with prior exposure to anti-TNF: results from the CORRONA registry. J. Rheumatol. 42(7), 10901098 (2015).

40. Forsblad-d'Elia H, Bengtsson K, Kristensen LE, et al. Drug adherence, response and predictors thereof for tocilizumab in patients with rheumatoid arthritis: results from the Swedish biologics register. Rheumatology (Oxford). 54(7), 1186-1193 (2015).

41. Gabay C, Riek M, Hetland ML, et al. Effectiveness of tocilizumab with and without synthetic diseasemodifying antirheumatic drugs in rheumatoid arthritis: results from a European collaborative study. Ann. Rheum. Dis. 75(7), 1336-1342 (2016).

42. Haraoui B, Jovaisas A, Bensen WG, et al. Use of corticosteroids in patients with rheumatoid arthritis treated with infliximab: Treatment implications based on a real-world Canadian population. RMD. Open. 1(1), e000078 (2015).

43. Fujiwara M, Kita Y. Reexamination of the assessment criteria for rheumatoid arthritis disease activity based on comparison of the Disease Activity Score 28 with other simpler assessment methods. Mod. Rheumatol. 23(2), 260-268 (2013).

44. Medeiros MM, de Oliveira BM, de Cerqueira JV, et al. Correlation of rheumatoid arthritis activity indexes (disease activity score 28 measured with ESR and CRP, simplified disease activity index and clinical disease activity index) and agreement of disease activity states with various cut-off points in a Northeastern Brazilian population. Rev. Bras. Reumatol. 55(6), 477-484 (2015). 